\title{
REVIEW ARTICLE Serogroup W meningococcal disease: global spread and current affect on the Southern Cone in Latin America
}

\author{
R. ABAD ${ }^{1}$, E. L. LÓPEZ ${ }^{2}$, R. DEBBAG ${ }^{3}$ AND J. A. VÁZQUEZ ${ }^{1 *}$ \\ ${ }^{1}$ Reference Laboratory for Meningococci, Institute of Health Carlos III, Majadahonda, Spain \\ ${ }^{2}$ Sociedad Argentina de Infectología Pediátrica and Departamento de Medicina del Hospital de Niños \\ 'Dr Ricardo Gutiérrez', Buenos Aires, Argentina \\ ${ }^{3}$ Sanofi Pasteur Medical Department for Latin America, Buenos Aires, Argentina
}

Received 9 October 2013; Final revision 13 March 2014; Accepted 21 April 2014; first published online 15 May 2014

\section{SUMMARY}

Meningococcal serogroup W strains have been emerging throughout the current century with most of the isolates belonging to the sequence type (ST11)/electrophoretic type (ET37) clonal complex (ST11/E37 CC), particularly since the international outbreak following Hajj 2000. That outbreak appears to have triggered off that trend, contributing to the spread of W ST11/ET37 CC strains globally; however, local strains could be also responsible for increases in the percentage and/or incidence rates of this serogroup in some countries. More recently, unexpected increases in the percentage and incidence rate of $\mathrm{W}$ has been noticed in different countries located in the South Cone in Latin America, and W ST11/ET37 CC strains now appear as endemic in the region and an extensive immunization programme with tetravalent conjugate vaccine (covering serogroups $\mathrm{A}$, $\mathrm{C}, \mathrm{Y}$ and $\mathrm{W}$ ) has been recently implemented in Chile. It is difficult to ascertain whether we are observing the emergence of W ST11 CC strains in different geographical areas or whether the Hajj 2000 strain is still spreading globally. Several aspects of the evolution of that situation are analysed in this paper, reviewing also the implications in immunization programmes. Closely related with the analysis of this potential evolution, it will be very interesting to monitor the evolution of serogroup $\mathrm{W}$ in the African meningitis belt after implementation of the extensive immunization programme with serogroup A conjugate vaccine that is currently underway. More data about carriers, transmission, clonal lineages, etc. are needed for taking decisions (target groups, outbreak control, defining the extent, etc.) to adapt the response strategy with potential interventions with broad coverage vaccines against the emergent serogroup $\mathrm{W}$.

Key words: Meningococcal disease, meningococcus (N. meningitidis), molecular epidemiology.

\section{Introduction}

Dynamics of the Neisseria meningitidis serogroups

Neisseria meningitidis is defined as a bacterium which is part of the commensal flora of the human upper respiratory tract. However, some of the clonal lineages, can cause invasive disease, in both endemic and epidemic forms. Meningococci are capsulated bacteria. The antigenic properties of the capsular polysaccharide are the base of its classification into

\footnotetext{
* Author for correspondence: Dr J. A. Vázquez Reference Laboratory for Meningococci, Institute of Health Carlos III, Majadahonda, Spain.

(Email: jvazquez@isciii.es)
} 
serogroups. Twelve serogroups are now universally recognized, but only six $(\mathrm{A}, \mathrm{B}, \mathrm{C}, \mathrm{W}, \mathrm{Y}, \mathrm{X})$ are associated with almost all clinical cases worldwide $[1,2]$. Nevertheless, the epidemiology as well as the serogroup distribution is highly dynamic and changes in the prevalence of $\mathrm{Y}$ and $\mathrm{X}$ serogroups have been well documented worldwide in the last decades [1-5]. The monitoring, surveillance and study of the potential spread of those serogroups and how they could replace current serogroups is a major challenge in public health for the important implications for interventions with immunization programmes, etc.

Since 2000, similarly showing specific characteristics, serogroup $\mathrm{W}$ has been spreading. Several aspects of this evolution are analysed in this review, and potential implications for immunization programmes are reviewed.

\section{Annual Hajj pilgrimages of 2000 and 2001: first international $\mathrm{W}$ meningococcal outbreak}

\section{Background}

Until 2000, serogroup W was associated with few sporadic cases of meningococcal disease worldwide [6] and it was suggested that hypothetically this serogroup had little potential to cause invasive disease in either sporadic or small epidemics [7].

Continuing with the history of the evolution of W strains, between 1991 and 1995 Kwara et al. [8] analysed a small number of $\mathrm{W}$ strains isolated in The Gambia and Mali, two countries located in the African meningitis belt. The authors concluded that all strains were closely related, suggesting the potential of $\mathrm{W}$ isolates to cause epidemics in African countries. In addition, the authors showed a great ability to anticipate future epidemiological consequences from field observations concluding that $\mathrm{W}$ strains might have a great opportunity to spread at the time of the annual Hajj, considering the large number of pilgrims going to Saudi Arabia from countries in the African meningitis belt.

\section{The Hajj 2000 international outbreak}

An international emergency was reported following the annual Hajj season in Saudi Arabia in March 2000 [6, 9], leading to secondary clusters in Muslim communities around the world as pilgrims returned to their countries of origin, demonstrating the epidemic potential of this serogroup [10, 11]. More than $400 \mathrm{~W}$ invasive cases were confirmed worldwide among pilgrims and their close contacts in at least 16 countries (Belgium, Denmark, Finland, France, Germany, Indonesia, Kuwait, Morocco, Norway, Oman, Saudi Arabia, Singapore, Sweden, The Netherlands, UK, USA) [6, 9] (Fig. 1).

\section{Characterization of Hajj 2000-associated W strains}

Different phenotypic and genotypic approaches were used to characterize the $\mathrm{W}$ strains isolated during the outbreak in an effort to determine the relationship between the different isolates and determine whether a single MenW clone was involved. Following this approach the Hajj 2000 outbreak was seen to be associated with $\mathrm{W}$ strains belonging to the sequence type (ST11)/electrophoretic type (ET37) clonal complex (ST11/ET37 CC), with very homogeneous phenotypic [serotype 2a, PorA variable region (VR) VR1-5, VR2-2] and genetic characteristics [6]. An intensive analysis using several molecular markers [pulsed-field gel electrophoresis (PFGE), multi-enzyme electrophoresis (MEE) or 16S rRNA gene sequencing] proved that the W strain associated with Hajj 2000 had been isolated in sporadic cases at least since 1970 in Africa and other continents [6]. In addition, a W strain belonging to the ST11/ET37 CC was also isolated from a patient from Indonesia who had just returned from Hajj 1996, suggesting that this clone had been circulating and associated with sporadic cases during the Hajj before 2000 [9].

Why was the strain circulating for years and potentially associated with the pilgrimage to Saudi Arabia before 2000 with no epidemiological relevance except sporadic cases? Did something change in the strains to improve their transmission capacities? Did something change in the environment or the host? There is no clear explanation to solve these questions and they will probably never be fully answered.

\section{How did the situation evolve after Hajj 2000?}

A significant number of $\mathrm{W}$ cases were reported towards the end of the 2001 epidemic season in Burkina Faso and Niger [12] and in January 2002 the first major epidemic of meningococcal disease caused by serogroup W began in Burkina Faso, with more than 12000 cases and 1400 deaths [13], confirming the potential of $\mathrm{W}$ isolates to cause major epidemics in African countries as suggested by Kwara et al. in 1998 [8] (Fig. 1). 

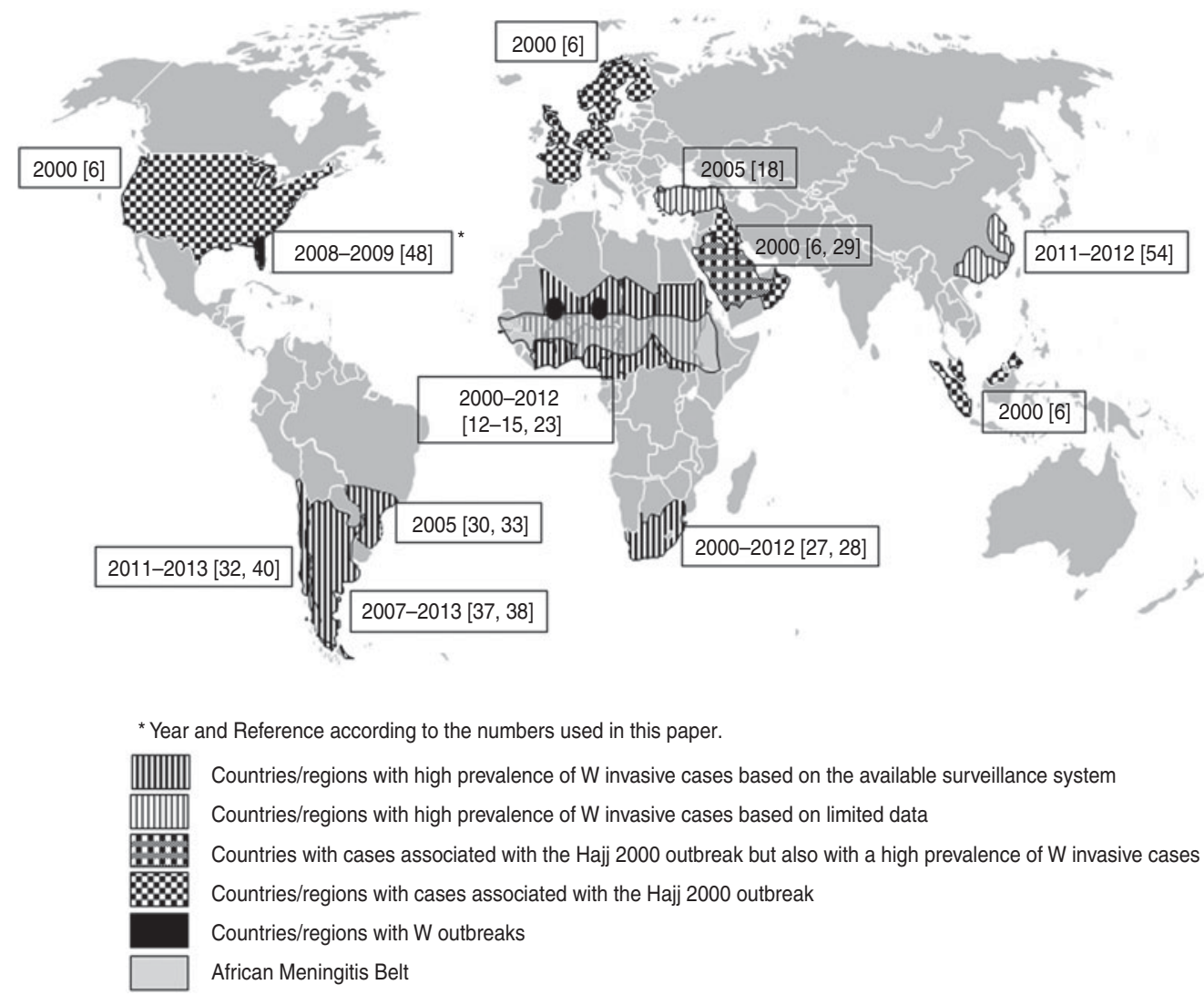

Fig. 1. World map showing the extent of known cases associated with serogroup $\mathrm{W}$ in countries reporting a significant proportion of cases and outbreaks.

This association with major epidemics suggests a high capability for transmission. In fact, consistent high levels of asymptomatic carriage of W ST11/ ET37 strains $(5 \cdot 5 \%$ prevalence in children aged 5 years) were found in 1996 during a study in children in The Gambia [14] and also recently in Burkina Faso [15], suggesting the capability for that clone both to persist and to be transmitted among susceptible populations. Wilder-Smith et al. [16] found that $15 \%$ of returning pilgrims to Hajj 2000 carried a single $\mathrm{W}$ clone and $55 \%$ were still carriers 6 months later, emphasizing that W ST11/ET37 CC strains are highly transmissible and persistent, thus constituting a potential risk not only to their close contacts but also for the community in general. These data were later confirmed by Nicolas et al. in 2005 [17], who analysed carriage of this clone in pilgrims and their family contacts in Morocco, Oman and Sudan, with W strains persisting for several months. More recently, a study conducted in Turkey in pilgrims travelling to the Hajj shows an important prevalence of $\mathrm{W}$ strains among carriers (around 11\%) before the trip and a significant percentage of acquisition of the epidemic strain $\mathrm{W}$ when they returned from the pilgrimage, also suggesting a high rate of transmission among their household contacts [18]. The duration and high prevalence of serogroup $\mathrm{W}$ in carried strains might favour the dissemination to the population in general (not only to the household contacts), resulting in an increasingly wider reservoir.

Owing to the observed high persistence and transmissibility, new reports of the increase and spread of W strains have appeared in literature $[5,19,20]$. In a recent study, W ST11/ET37 CC strains were identified in almost all the countries in the African meningitis belt from 2004 to 2010, mainly associated with sporadic cases and small epidemics [5]. The W ST11/ ET37 CC strains seemed to disappear from Burkina Faso after 2006 and the sporadic $\mathrm{W}$ isolates found in this country belonged to ST175 CC, which has not been associated with major epidemics and is responsible for endemic disease cases. However, W ST11/ET37 CC re-emerged in carriers as well as patients, after the country-wide mass vaccination with A conjugate vaccine [15]. In Chad serogroup W strains became predominant during most of the period 
2004-2008, where the strains belonged to either ST11/ ET37 CC or ST175 CC. This shows the contribution of other CCs in serogroup W emergence in Africa and other geographical regions, suggesting the continuous emergence of new $\mathrm{W}$ genetic lineages [11]. An increased number of $\mathrm{W}$ cases was observed in Niger in 2010 and the isolates belonged to ST11/ ET37 CC [21]. The study shows a continuous spread of W strains in the African meningitis belt. Whether the introduction of a new serogroup A conjugate vaccine (PsA-TT) [22] in a nationwide mass vaccination campaign in the African meningitis belt starting in Burkina Faso in December 2010 will have some effect in other non-A serogroup epidemic strains, particularly in W strains, is something to be evaluated in future studies. Looking at the current situation, during the first quarter of 2012 [23], alerts for meningococcal disease were reported in 10 countries of the African meningitis belt (Fig. 1). The outbreaks were mainly associated with $\mathrm{W}$ strains. In spite of tremendous success in controlling epidemics for serogroup A in the region [24, 25], enhanced surveillance needs to be maintained to monitor the evolution of serogroups in the region both in patients and in asymptomatic carriers. There is no evidence for serogroup replacement with serogroup $\mathrm{C}$ conjugate vaccines, but this is not demonstrated for other serogroups like A or W. Recent studies show a strong impact of MenAfriVac vaccination on serogroup A in carriers $[15,26]$ and there is not yet strong evidence for serogroup replacement [15], in either the disease or its carriers.

In South Africa, a country located outside the African meningitis belt, serogroup $\mathrm{W}$ was established as the predominant cause of endemic disease in 2008 [27]. This situation currently looks rather stable [28] and is associated with a high mortality rate. A similar situation, with serogroup $\mathrm{W}$ appearing as predominant and becoming endemic, has been observed in Saudi Arabia, where the tetravalent conjugate vaccine has been recommended in the National Immunization Schedule for children at the age of 9 months since 2012 [29] underlining the need for flexible and current public health interventions.

\section{The travel of W strains to the South Cone in Latin America: spreading and becoming endemic in some countries}

Although there have been no reports of serogroup W cases from Latin American countries associated with
Hajj 2000 [6, 9], this may be due to the absence of well-established surveillance for invasive meningococcal disease (IMD) in many countries in the region. A hypothetical association with the Muslim communities located in the region is no more than speculation because, as has been mentioned, there is no epidemiological information to establish that association. However, the emergence of serogroup W meningococcal cases has been observed in the South Cone (a geographical region composed of the southernmost areas of South America, south of the Tropic of Capricorn, including Argentina, Southern Brazil, Chile, Paraguay, Uruguay) in the last decade. This geographical area seems to be, for now, the last stage of the spread of serogroup W. The trends observed in the region suggest that serogroup W might become endemic in some countries of the region. This observation is a large change compared to with past situations (with serogroup $\mathrm{W}$ just associated with large epidemics or local outbreaks), highlighting the need to consider the inclusion of this serogroup in any vaccination strategy for the region.

\section{Brazil: the first country with reports}

An increase in the percentage of IMD cases associated with serogroup $\mathrm{W}$ has been evident in Rio Grande do Sul (incidence rate for IMD $1.5 \times 10^{5}$ ), in the south of Brazil, at least since 2004 [30], with increases of this serogroup also reported in Rio de Janeiro [31]. In fact, in the South, W strains represented $3 \cdot 2 \%$ during the period $1995-2003$, rising to $17 \cdot 8 \%$ in $2003-2005$ [32], the estimated current frequency of $\mathrm{W}$ for Brazil is $5 \cdot 8 \%$ (incidence rate for IMD around $2 \times 10^{5}$ ). Although W strains are characterized as ST11/ET37 $\mathrm{CC}$ with similar phenotypic characteristics as the isolates associated with Hajj 2000 [30, 33], it has been suggested that they represent local isolates of ST11/ ET37 CC rather than strains clearly evolving from those isolated during Hajj 2000, as described in some other countries [11,34]. An increased frequency of serogroup W in Brazil has been observed with no reduction in the total number of IMD cases over time, suggesting that the change in the percentage of serogroup $\mathrm{W}$ cases is not due simply to a decrease in cases by other serogroups.

\section{Argentina: $W$ strains become predominant}

In Argentina, a country adjacent to Brazil, serogroup $\mathrm{W}$ represented less than $6 \%$ of the confirmed cases up to 2006 , rising to $13 \%$ in 2007 and represented around 
50\% from 2008 onwards [35]. In 2005 and 2006 an unexpected cluster of cases was observed in Misiones, a northern province on the border with Paraguay and Brazil, which might represent the beginning of the new situation. After that, clinical cases associated with $\mathrm{W}$ appeared mainly in the Buenos Aires province in 2007 and 2008, spreading to the rest of the country later [36]. Preliminary and limited data showed those $\mathrm{W}$ strains isolated in Argentina as belonging to ST11/ET37 CC with similar characteristics to those found for the Hajj 2000 strains [37]. A recent report by Sorhouet-Pereira et al. [38] confirmed the presence and prevalence of the epidemic clone $\mathrm{W}: 2 \mathrm{a}: \mathrm{P} 1 \cdot 5,2$ : ST11 in the country, but the molecular characterization is insufficient to determine whether the situation is similar to that found in Brazil, with local isolates belonging to the same epidemic clone or whether the Argentinean strains are closely related to the Hajj 2000 outbreak strain. This distinction is important as it may lead to the conclusion either that we are observing the emergence of W ST11 CC strains in different geographical areas, or that the Hajj 2000 strains are still spreading globally. In Argentina the incidence rate of IMD has been around 0.6/100000 inhabitants from 2005 to date [38]. Thus, the increase proportion of $\mathrm{W}$ strains is not associated with increases in the number of cases of IMD in this country, but it is presumably not an artefact due to a decrease in the number of cases related to other serogroups.

\section{Chile: increases in percentages, incidence and mortality rates}

Some increases in the percentage of $\mathrm{W}$ were also observed in 2009 and 2010 in Uruguay, Paraguay and Chile [35], all located in the South Cone in Latin America. Trends observed in Chile were recently confirmed with a large increase in the number of $\mathrm{W}$ cases in 2011 and particularly in 2012 reported by the Ministry of Health and an increase in the mortality rate, to $25 \%$ in cases associated with W $[32,39]$. The increase was particularly important in children aged $<5$ years [40] but was significant in all age groups. In light of a higher percentage of $\mathrm{W}$ cases also associated with an increase in the incidence rate of IMD $\left(0 \cdot 8 \times 10^{5}\right.$ in 2012 compared with 0.4 in 2011 or 0.5 in 2010), particularly in and around the capital city, the tetravalent $(\mathrm{A}+\mathrm{C}+\mathrm{Y}+\mathrm{WW})$ conjugate vaccine was administered throughout the country from the end of 2012, with children aged between 9 months and 4 years the target population [41]; this intervention achieved a high coverage. The immunization programme is still ongoing with new cohorts being vaccinated. Eighty-seven cases of IMD associated with serogroup W were reported in Chile in 2013 and there were only two cases in the vaccinated age group corresponding to two children who did not receive the vaccine [42]. The number of $\mathrm{W}$ cases in 2013 is significantly higher than in 2012 when 60 $\mathrm{W}$ cases were reported, suggesting that other age groups should be targeted for new interventions with tetravalent vaccines in order to exercise control on the situation, as we learned in the past with the use of $\mathrm{C}$ conjugate vaccines [43]. In this sense, a carrier survey including more than 4000 subjects aged between 11 and 19 years was performed in the country and the data will be very useful for implementing strategies for immunization in the future [44]. The molecular characterization of a limited number of $\mathrm{W}$ strains isolated in Chile during 2010 and 2011 shows that most of the W strains belong to ST11/ET37 CC [40], but other CCs are also appearing, particularly in 2011. Those additional CCs identified in 2011 were those usually associated with serogroup B strains, suggesting the possibility of switching events in the meningococcal population in Chile. A recent report [45] shows that there were nine different band patterns after analysis of DNA by PFGE in $37 \mathrm{~W}$ strains (with $57 \%$ belonging to only one band pattern profile), with a similar level of variability to that previously observed for the ST11/ET37 clonal lineage [11]. These data suggest a continuous diversification of the W ST11/ET37 CC with new variants being associated with clinical cases. It is very important to monitor the evolution of the situation carefully not only in terms of the efficacy of the intervention but also analysing the immune response (magnitude, duration), etc. This experience will be very useful to confront potential similar situations in the future in other countries.

\section{Tracking the expansion of $\mathbf{W}$ strains: molecular characterization}

As mentioned elsewhere in this review, many studies have been made characterizing $\mathrm{W}$ isolates both phenotypically and genetically in an effort to get a better understanding of the evolution, not only of serogroup W but particularly of those strains belonging to ST11/ET37 CC (Table 1). Genetic drift should be driving the evolution of the Hajj-related strains after the 2000 outbreak. High rates of W strains in carriers 
Table 1. Methods used for characterization of $W$ strains in different studies found in the literature

\begin{tabular}{|c|c|c|c|c|c|c|c|c|c|c|c|c|c|}
\hline Ref.* & $\begin{array}{l}\text { No. of } \\
\text { W strains }\end{array}$ & Period of time & Country & Serotype & Serosubtype & Genosubtype & MLST (CC) & MEE & PFGE & MLDF & $16 \mathrm{~S}$ & fetA & WGS \\
\hline [6] & 76 & $1970-2000$ & Worldwide & $\sqrt{ }$ & $\sqrt{ }$ & $\sqrt{ }$ & $\sqrt{ }$ & $\sqrt{ }$ & $\sqrt{ }$ & $\sqrt{ }$ & $\sqrt{ }$ & & \\
\hline [33] & 216 & 1990-2005 & Brazil & $\sqrt{ }$ & $\sqrt{ }$ & $\sqrt{ }$ & $\sqrt{ }$ & & $\sqrt{ }$ & & $\sqrt{ }$ & $\sqrt{ }$ & \\
\hline [8] & 8 & 1994-1995 & The Gambia/Mali & $\sqrt{ }$ & $\sqrt{ }$ & & & $\sqrt{ }$ & $\sqrt{ }$ & & & & \\
\hline [11] & 205 & 1994-2002 & France & $\sqrt{ }$ & $\sqrt{ }$ & & $\sqrt{ }$ & & $\sqrt{ }$ & $\sqrt{ }$ & & & \\
\hline [9] & 4 & 2000 & USA & $\sqrt{ }$ & $\sqrt{ }$ & $\sqrt{ }$ & & $\sqrt{ }$ & & & $\sqrt{ }$ & & \\
\hline [28] & 406 & $2000-2005$ & South Africa & & & & $\sqrt{ }$ & & $\sqrt{ }$ & & & & \\
\hline [13] & 131 & 2002 & Burkina Faso & $\sqrt{ }$ & $\sqrt{ }$ & & $\sqrt{ }$ & & & & & & \\
\hline [30] & 14 & 2003-2005 & South of Brazil & & & $\sqrt{ }$ & $\sqrt{ }$ & & & & & & \\
\hline [37] & 7 & 2007-2008 & Argentina & $\sqrt{ }$ & & $\sqrt{ }$ & $\sqrt{ }$ & & & & & $\sqrt{ }$ & \\
\hline [5] & 105 & 2004-2010 & Men. belt (Africa) & & & $\sqrt{ }$ & $\sqrt{ }$ & & & & & $\sqrt{ }$ & \\
\hline [48] & 14 & 2008-2009 & Florida (USA) & & & & $\sqrt{ }$ & & $\sqrt{ }$ & & & & \\
\hline [38] & 65 & 2010 & Argentina & $\sqrt{ }$ & $\sqrt{ }$ & & $\sqrt{ }$ & & & & & & \\
\hline [40] & 25 & 2010-2011 & Chile & & & $\sqrt{ }$ & $\sqrt{ }$ & & & & & & \\
\hline [47] & 14 & 2012 & France/Ivory Coast & $\sqrt{ }$ & & $\sqrt{ }$ & $\sqrt{ }$ & & & & & $\sqrt{ }$ & \\
\hline [54] & 11 & 2011-2012 & China & & $\sqrt{ }$ & & $\sqrt{ }$ & & $\sqrt{ }$ & & & & \\
\hline [53] & 10 & 2003-2012 & South Africa & - & - & - & - & - & - & - & - & - & $\sqrt{ }$ \\
\hline
\end{tabular}

MLST, Multi-locus sequence type; CC, clonal complex; MEE, multi-enzyme electrophoresis; PFGE, pulsed-field gel electrophoresis; MLDF, multi-locus DNA fragment typing; WGS, whole genome sequence.

* Reference according to the numbers used in this paper. 
and persistence among them allows the $\mathrm{W}$ strains to evolve and, emerging genetic variants hinder analysis of the evolution of the epidemic strains. In this sense fine characterization of $\mathrm{W}$ isolates is critical for tracking their spread and following the evolution.

However, the level of characterization already used in different studies is rather heterogeneous and it is difficult to compare findings among them (Table 1). Most of the authors define at least the genosubtype (PorA variable regions) and sequence type; then all strains characterized as $\mathrm{P} 1 \cdot 5,2$ belonging to the ST11/ET37 CC are referred to as Hajj 2000 or closely related strains [37, 38, 40, 46]. However, the inclusion of additional markers shows that several genetically related subgroups are present in W ST11/ET37 CC, suggesting that fine characterization is needed to fully understand trends in transmission and spread [1, 5, 11]. Only Mayer et al. in 2002 [6] compared the use of two phenotypic (serotyping and serosubtyping) and six molecular markers [16S, PFGE, MEE, VR-1 VR-2, multi-locus sequence typing (MLST), multi-locus DNA fragment typing (MLDF)] for characterization of $\mathrm{W}$ strains, defining sensitivity, specificity, positive predictive value (PPV) and negative predictive value (NPV) for all molecular subtyping methods for identifying the Hajj outbreak-associated strains. The authors concluded that $16 \mathrm{~S}$ sequencing, PFGE, and MEE provided the best specificity $(98 \%$, $86 \%, 80 \%$, respectively) and PPV (96\%, 79\%, 72\%, respectively), and a combination of PFGE and MEE resulted in a specificity of $90 \%$ and a PPV of $84 \%$. The sequence of fet $A$ variable region has also been used later, but there is no specific analysis of its specificity and/or PPV values [37, 47]. Some authors have shown that other genetic lineages among $\mathrm{W}$ strains (ST2495 and ST22) might be expanding, contributing to the genetic diversification of the meningococci associated with serogroup $\mathrm{W}$ [11]. $N$. meningitidis is subject to horizontal DNA transfer and re-assortment of genes between different strains producing genetic variants. Therefore, characterization of invasive meningococcal strains by several fine molecular typing approaches is essential to monitor and understand the evolution of the situation. As an example, there was a cluster of W cases in southeastern Florida [48], in which the authors used PFGE and MLST for characterization of the isolates (7/12), with all $\mathrm{W}$ strains belonging to ST11 CC with an indistinguishable (or closely related) band pattern profile by PFGE. With this microbiological information the authors suggest that 'The dominant clonal complex observed in the Florida cluster matches the dominant type recently observed in Argentina and Brazil, raising the possibility of introduction to Southeastern Florida from South America.' However, at that time there was no information about PFGE patterns in $\mathrm{W}$ strains isolated in the South Cone in Latin America, so we only know that the strains isolated in both Florida and the South Cone belonged to ST11 CC which is not enough to support the suggestion. A minimal consensus about what molecular markers should be used might help to be able to compare definitely different findings in different geographical areas and reach a conclusion as to whether we are observing the emergence of W ST11 CC strains in different geographical areas or, on the contrary, the Hajj 2000 strains are still spreading globally.

\section{W ST11/ET37 CC strains: where do they come from?}

Because of the sporadic isolation of ST11/ET37 W strains in different geographical locations and years, it has been suggested that the 2000 outbreak associated with the Hajj might have been the result of spreading of a pre-existing, although not well known, W ST11/ET37 CC strain, after an amplification effect possibly enhanced by favourable environmental circumstances [6], rather than emergence of a new $\mathrm{W}$ strain.

Immunological pressure might have had an important role in this history. The annual Muslim pilgrimage to Saudi Arabia had been previously associated with outbreaks of meningococcal disease in returning pilgrims [49]. After the first pandemic of meningococcal serogroup A infection during Hajj 1987, Saudi Arabian authorities required vaccination with a bivalent serogroups $\mathrm{A}+\mathrm{C}$ vaccine for all pilgrims arriving in the country [50]. Since the mid-1990s, because of that visa requirement for all pilgrims entering the country, millions of doses mainly of meningococcal $\mathrm{A}+\mathrm{C}$ polysaccharide vaccine have been administered (although some countries were using the quadrivalent meningococcal $\mathrm{A}+\mathrm{C}+\mathrm{W} 135+\mathrm{Y}$ polysaccharide vaccine), allthough those $A+C$ polysaccharide vaccines offer no protection from W strains. Since 2002 the quadrivalent meningococcal vaccine $(\mathrm{A}+\mathrm{C}+\mathrm{Y}+\mathrm{W})$ has been required to obtain the Hajj visa.

Similarities in both molecular and phenotypic markers have been suggested when comparing W with serogroup C strains, both belonging to ST11/ ET37 CC $[6,51]$. In addition to being a member of 
the ST11/ET37 complex, additional molecular and phenotypic markers of this $\mathrm{W}$ clone are very similar to those of serogroup C strains of the ST11/ET37 complex that are associated with outbreaks and sporadic cases of meningococcal disease. The question is whether W ST11/ET37 CC strains have derived from serogroup $\mathrm{C}$ strains of the same $\mathrm{CC}$ through a capsular-switching event, often described in meningococcal strains involving different serogroups [52] or both W and C ST11/ET37 CC represent two separate and distinct evolutionary lines with a distant common ancestor that subsequently differentiated into different serogroups. The data already discussed in this review and presented in several studies show that an increasing numbers of $\mathrm{W}$ strains were reported in Saudi Arabia every year from 1990 to 2000 [6, 51] suggesting some circulation of $\mathrm{W}$ strains before Hajj 2000. This finding is confirmed by the isolation of a similar W strain in a patient from Indonesia who was a pilgrim returning from the 1996 Hajj. Additionally, very similar strains have been recovered worldwide at least since 1970 [6], suggesting that the hypothetical capsular-switching event (from $\mathrm{C}$ to $\mathrm{W}$ or in the opposite direction) took place possibly before 1970 [6].

Carriers, transmission and persistence data already commented upon in this review [14-18, 25, 26] might indicate that W ST11/ET37 CC strains appear to be significantly more transmissible than C ST11/ET37 $\mathrm{CC}$ isolates and it has been suggested that the modification of this capability might be related to the serogroup switch [26]. However, this different characteristic might also suggest that in fact $\mathrm{W}$ and C ST11/ET37 CC represent two separate and distinct evolutionary lines among the ST11/ET37 CC.

The question then is whether this clone has the potential to settle in the population maintaining adequate rates of transmission to be associated with endemic or epidemic situations or whether the situation at the beginning of this century is a one-off and temporary. A recent report shows recent transmission of W ST11/ET37 CC in southeastern China [54], suggesting that the strains might be still globally spreading. The global distribution of reports of $\mathrm{W}$ increases is shown in Figure 1 and it might only reflect part of the extension of serogroup $\mathrm{W}$, and those geographical areas with no apparent effect could be associated with poor surveillance systems. How this situation evolve in the next future is one of the most important topics for those people involved in prevention and control of meningococcal disease worldwide.

\section{Conclusions}

In the light of the data found in the literature it can be concluded that:

- Serogroup W has been emergent during the current century, with strains belonging to ST11/ET37 CC being most prevalent among the serogroup. However, genetic variants and other clonal lineages might be contributing to the emergence.

- The outbreak in 2000 associated with W strains appears to have triggered off that trend, contributing to the spread of W ST11/ET37 CC strains globally. However, local strains might be also responsible for the increased percentage of this serogroup.

- An unexpected increase in the percentage of W has been observed in different countries located in the South Cone in Latin America. W ST11/ET37 CC strains now appear as endemic in the region and a large immunization programme with tetravalent conjugate vaccine has been implemented in Chile.

- It will be very interesting to monitor the evolution of serogroup $\mathrm{W}$ in the African meningitis belt after the implementation of an extensive immunization programme with serogroup A conjugate vaccine, and also trends in serogroup distribution in Chile after administration of the conjugate tetravalent vaccine. Reinforced surveillance is needed to evaluate potential future interventions with broad coverage vaccines against the emergent serogroup $\mathrm{W}$.

\section{DECLARATION OF INTEREST}

R.D. is employed by Sanofi-Pasteur; J.A.V. received grants for research from Sanofi-Pasteur, Novartis Vaccines, GlaxoSmithKline and Pfizer.

\section{REFERENCES}

1. Harrison LH, Trotter CL, Ramsay ME. Global epidemiology of meningococcal disease. Vaccine 2009; 27 (Suppl. 2): B51-B63.

2. Sáfadi MA, et al. The epidemiology of meningococcal disease in Latin America 1945-2010: an unpredictable and changing landscape. Epidemiology and Infection 2012; 9: 1-12.

3. Abad R, et al. Molecular characterization of invasive serogroup Y Neisseria meningitidis strains isolated in the Latin America region. Journal of Infection 2009; 59: 104-114.

4. Bröker M, et al. Increase of meningococcal serogroup $\mathrm{Y}$ cases in Europe: a reason for concern? Human Vaccines and Immunotherapeutics 2012; 8: 685-688. 
5. Caugant DA, et al. (2012). Molecular characterization of invasive meningococcal isolates from countries in the African meningitis belt before introduction of a serogroup A conjugate vaccine. PLoS One 2012; 7: e46019.

6. Mayer LW, et al. Outbreak of W meningococcal disease in 2000: not emergence of a new W strain but clonal expansion within the electrophoretic type-37 complex. Journal of Infectious Diseases 2002; 185: 1596-1605.

7. Griffiss JM, Brandt BL. Disease due to serogroup W Neisseria meningitidis. Pediatrics 1979; 64: 218-221.

8. Kwara A, et al. Meningitis caused by a serogroup W clone of the ET37 complex of Neisseria meningitidis in West Africa. Tropical Medicine and International Health 1998; 3: 742-746.

9. Popovic T, et al. Neisseria meningitidis serogroup W isolates associated with the ET37 complex. Emerging Infectious Diseases 2000; 6: 428-429.

10. Hahné SJ, et al. W meningococcal disease in England and Wales associated with Hajj 2000 and 2001. Lancet 2002; 359: 582-583.

11. Taha MK, et al. Continuing diversification of Neisseria meningitidis $\mathrm{W}$ as a primary cause of meningococcal disease after emergence of the serogroup in 2000. Journal of Clinical Microbiology 2004; 42: 4158-4163.

12. Taha MK, et al. Neisseria meningitidis serogroups W and A were equally prevalent among meningitis cases occurring at the end of the 2001 epidemics in Burkina Faso and Niger. Journal of Clinical Microbiology 2002; 40: 1083-1084.

13. Koumaré B, et al. The first large epidemic of meningococcal disease caused by serogroup W, Burkina Faso, 2002. Vaccine 2007; 25 (Suppl. 1), A37-41.

14. MacLennan JM, et al. Carriage of serogroup W, ET37 meningococci in the Gambia: implications for immunisation policy. Lancet 2000; 356: 1078.

15. Kristiansen PA, et al. Phenotypic and genotypic characterization of meningococcal carriage and disease isolates in Burkina Faso after mass vaccination with a serogroup a conjugate vaccine. BMC Infectious Diseases 2013; 13: 363.

16. Wilder Smith A, et al. Persistence of W Neisseria meningitidis carriage in returning Hajj pilgrims: risk for early and late transmission to household contacts. Emerging Infectious Diseases 2003; 9: 123-126.

17. Nicolas $\mathbf{P}$, et al. Pharyngeal carriage of serogroup W Neisseria meningitidis in Hajjees and their family contacts in Morocco, Oman and Sudan. APMIS 2005; 113: $182-186$.

18. Ceyhan M, et al. Acquisition of meningococcal serogroup W-135 carriage in Turkish Hajj pilgrims who had received the quadrivalent meningococcal polysaccharide vaccine. Clinical and Vaccine Immunology 2013; 20: 66-68.

19. Wilder-Smith A, Chow A, Goh KT. Emergence and disappearance of $\mathrm{W}$ meningococcal disease. Epidemiology \& Infection 2009; 138: 976-978.

20. Parent du Châtelet I, Barboza P, Taha MK. W invasive meningococcal infections imported from
Sub-Saharan Africa to France, January to April 2012. Eurosurveillance 2012; 17: pii=20181.

21. Collard JM, et al. Increase in Neisseria meningitidis serogroup W, Niger, 2010. Emerging Infectious Diseases 2010; 16: 1496-1498.

22. Frasch CE, Preziosi MP, LaForce FM. Development of a group A meningococcal conjugate vaccine, MenAfriVac ${ }^{\mathrm{TM}}$. Human Vaccines and Immunotherapeutics 2012; 8: 715-724.

23. World Health Organization. Meningococcal disease: situation in the African Meningitis Belt (http://www. who.int/csr/don/2012_05_24/en/index.html). Accessed 28 July 2013

24. Djingareya MH, et al. Effectively introducing a new meningococcal A conjugate vaccine in Africa: The Burkina Faso experience. Vaccine 2012; 30 (Suppl. 2): B40-B45.

25. Daugla DM, et al. Effect of a serogroup A meningococcal conjugate vaccine (PSA-TT) on serogroup A meningococcal meningitis and carriage in Chad: a community trial. Lancet. Published online 11 September 2013. doi:10.1016/S0140-6736(13)61612-8.

26. Kristiansen PA, et al. Impact of the serogroup A meningococcal conjugate vaccine, MenAfriVac, on carriage and herd immunity. Clinical Infectious Diseases 2013; 56: $354-363$.

27. von Gottberg A. Neisseria meningitidis. Communicable Diseases Surveillance Bulletin 2012; 10: 60-63.

28. von Gottberg A, et al. Emergence of endemic serogroup $\mathrm{W}$ meningococcal disease associated with a high mortality rate in South Africa. Clinical Infectious Diseases 2008; 46: 377-386.

29. Ministry of Health Portal. Kingdom of Saudi Arabia. Basic vaccination schedule (http://www.moh.gov.sa/en/ HealthAwareness/EducationalContent/HealthTips/Pages/ Tips-005.aspx). Accessed 26 July 2013.

30. Weidlich L et al. High prevalence of Neisseria meningitidis hypervirulent lineages and emergence of W:P1·5,2: ST11 clone in Southern Brazil. Journal of Infection 2008; 57: 324-331.

31. Barroso DE, Rebelo MC. Recognition of the epidemiological significance of Neisseria meningitidis capsular serogroup W in the Rio de Janeiro region, Brazil. Memórias do Instituto Oswaldo Cruz 2007; 102: 773-775.

32. López EL, Debbag R. Meningococcal disease: always present. Serogroup changes in the Southern Cone. Revista Chilena de Infectología 2012; 29: 587-594.

33. Lemos AP, et al. Phenotypic and molecular characterization of invasive serogroup W Neisseria meningitidis strains from 1990 to 2005 in Brazil. Journal of Infection 2009; 60: 209-217.

34. Ferreira E, et al. Neisseria meningitidis serogroup W in Portugal-presence of the ST11/ET37 clonal complex. Pathologie Biologie 2007; 56: 94.

35. Ibarz-Pavón AB, et al. Laboratory-based surveillance of Neisseria meningitidis isolates from disease cases in Latin American and Caribbean countries, SIREVA II 2006-2010. PLoS One 2012; 7: e 44102.

36. Efron AM, et al. Significant increase of serogroup W invasive Neisseria meningitidis strains in Argentina: a 
new epidemiological feature on the region. Sixteenth International Pathogenic Conference 2008, Rotterdam, The Netherlands. P-169. (http://neisseria.org/ipnc/2008/ Abstracts_poster_presentations_IPNC_2008.pdf). Accessed 28 July 2013.

37. Efron AM, et al. W invasive meningococcal strains spreading in South America: significant increase in incidence rate in Argentina. Journal of Clinical Microbiology 2009; 47: 1979-1980.

38. Sorhouet-Pereira C, et al. Phenotypic and genotypic characteristics of Neisseria meningitidis diseasecausing strains in Argentina, 2010. PLoS One 2013; 8: e 58065 .

39. Ministerio Salud Pública Republica de Chile. Situation of meningococcal disease caused by serogroup W (http://epi.minsal.cl/epi/html/bolets/reportes/Meningitis/ W_Web_SE46A.pdf) Accessed 26 July 2013.

40. Barra GN, et al. Molecular characterization of invasive Neisseria meningitidis strains isolated in Chile during 2010-2011. PLoS One 2013; 8: e 66006.

41. Ministerio Salud Pública Republica de Chile. Action Plan W (http://www.minsal.gob.cl/portal/url/ page/minsalcl/g_varios/boletin/meningitis/page_1.html). Accessed 26 July 2013.

42. Ministerio Salud Pública Republica de Chile. Meningococcal disease (CIE-10: A39). Epidemiological situation, January-December, 2013. Action Plan W-135 (http://epi.minsal.cl/epi/html/AtlasInteractivos/AtlasBET/ ABET_04/EM_BET4.pdf) Accessed 22 January 2014.

43. Cohn AC, Harrison LH. Meningococcal vaccines: current issues and future strategies. Drugs 2013; 73: 1147-1155.

44. Ministerio Salud Pública Republica de Chile. Research on carriage of Neisseria meningitidis (http:// web.minsal.cl/portal/url/page/minsalcl/g_varios/boletin/ meningitis/page_6.html. Accessed 22 January 2014.
45. Instituto de Salud Pública de Chile. Report of the results of laboratory surveillance. invasive disease. Neisseria meningitidis 2012. (http://www.ispch.cl/sites/default/ files/Informe $\% 20$ N.m\%202012.pdf). Accessed 26 July 2013.

46. Taha MK, et al. Serogroup W meningococcal disease in Hajj pilgrims. Lancet 2000; 356: 2159.

47. Taha MK, et al. Travel-related Neisseria meningitidis serogroup W135 infection, France. Emerging Infectious Diseases 2013; 19: 1030-1032.

48. Doyle TJ, et al. Cluster of serogroup W135 meningococci, southeastern Florida, 2008-2009. Emerging Infectious Diseases 2010; 16: 113-115.

49. Jones DM, Sutcliffe EM. Group A meningococcal disease in England associated with the Hajj. Journal of Infection 1990; 21: 21-25.

50. Al-Gahtani YM, et al. Epidemiological investigation of an outbreak of meningococcal meningitis in Makkah (Mecca), Saudi-Arabia, 1992. Epidemiology \& Infection 1995; 115: 399-409.

51. Wang JF, et al. Antigenic and epidemiologic properties of the ET37 complex of Neisseria meningitidis. Journal of Infectious Diseases 1993; 167: 1320-1329.

52. Castilla J, et al. B:2a:P1.5 meningococcal strains likely arisen from capsular switching event still spreading in Spain. Journal of Clinical Microbiology 2009; 47: 463-465.

53. du Plessis M, et al. Whole genome resolution of selected Neisseria meningitidis serogroup W strains, South Africa, 2003-2012. 12th EMGM 2013, Bad Loipersdorf, Austria. P-097 (http://emgm.eu/meetings/emgm2013/ Abstract_Book_EMGM_2013.pdf). Accessed 28 July 2013.

54. Zhou H, et al. Spread of Neisseria meningitidis serogroup W clone, China. Emerging Infectious Diseases 2013; 19: 1496-1469. 